\title{
Fine-Grained Protein Fold Assignment by Support Vector Machines Using Generalized $n$ Peptide Coding Schemes and Jury Voting From Multiple-Parameter Sets
}

\author{
Chin-Sheng Yu, ${ }^{1}$ Jung-Ying Wang, ${ }^{2}$ Jinn-Moon Yang, ${ }^{1}$ Ping-Chiang Lyu, ${ }^{3}$ Chih-Jen Lin, ${ }^{2 *}$ and Jenn-Kang Hwang ${ }^{1 *}$ \\ ${ }^{1}$ Department of Biological Science and Technology, National Chiao Tung University, Hsin Chu, Taiwan \\ ${ }^{2}$ Department of Computer Science, National Taiwan University, Taipei, Taiwan \\ ${ }^{3}$ Department of Life Sciences, National Tsing Hua University, Hsin Chu, Taiwan
}

\begin{abstract}
In the coarse-grained fold assignment of major protein classes, such as all- $\alpha$, all- $\beta, \alpha+$ $\beta, \alpha / \beta$ proteins, one can easily achieve high prediction accuracy from primary amino acid sequences. However, the fine-grained assignment of folds, such as those defined in the Structural Classification of Proteins (SCOP) database, presents a challenge due to the larger amount of folds available. Recent study yielded reasonable prediction accuracy of $56.0 \%$ on an independent set of 27 most populated folds. In this communication, we apply the support vector machine (SVM) method, using a combination of protein descriptors based on the properties derived from the composition of $\boldsymbol{n}$-peptide and jury voting, to the fine-grained fold prediction, and are able to achieve an overall prediction accuracy of $69.6 \%$ on the same independent set-significantly higher than the previous results. On 10-fold cross-validation, we obtained a prediction accuracy of 65.3\%. Our results show that SVM coupled with suitable global sequence-coding schemes can significantly improve the fine-grained fold prediction. Our approach should be useful in structure prediction and modeling. Proteins 2003;50:531-536. $\odot 2003$ Wiley-Liss, Inc.
\end{abstract}

Key words: support vector machines; fine-grained fold prediction; global sequence-coding scheme; $n$-peptide

\section{INTRODUCTION}

As a result of the progress in experimental genomics, tremendous amounts of sequence data have emerged, and the increase in the number of putative protein sequences greatly exceeds that of three-dimensional (3D) structures of proteins. Hence, to extract 3D structures from sequences becomes even more important today. Roughly speaking, there are generally two kinds of approaches to structure prediction. ${ }^{1}$ One is the ab initio method that predicts structures directly from the sequences based on the general physicochemical principles. ${ }^{2-7}$ The other is the empirical method that relies on the empirical knowledge of proteins structures or sequences to assign the query sequences to the proper folds by either homology modeling, threading techniques, or a taxonometric approach. ${ }^{8-13}$ Homology modeling identifies the possible template structures of the query sequences by aligning them with the sequences of known 3D structures, based on the criterion that proteins with sequence identity higher than $25 \%$ usually have similar structures. Threading techniques find the possible folds by the sequence-structure alignment, without relying on the sequence homology between the query and target sequences. The taxonometric method, based on the assumption that the number of folds is limited, tries to predict protein structures in terms of the assignment of query sequences to the particular classification of protein folds. Proteins are said to have a common folding structure if their major secondary structures have similar arrangement and topologic connections. The latter approach becomes increasingly important as a result of the fast growth of protein structures. Previous studies ${ }^{11,14-16}$ have shown that in coarse-grain structural classification such as all- $\alpha$, all- $\beta, \alpha+\beta, \alpha / \beta$, and irregular folds, ${ }^{17}$ one can easily achieved $70 \%$ or better prediction accuracy from the amino acid composition. However, in order to obtain a high-resolution $3 \mathrm{D}$ structure, one needs to be able to assign fine-grained folds for the query structures. The assignment of fine-grained folds, such as that defined in the Structural Classification of Proteins (SCOP) database, presents a challenge for structure prediction due to the larger number of folds. Recently, Ding and Dubchak ${ }^{13}$ applied support vector machines (SVMs) to the problem of fold assignment. They used six coding schemes ${ }^{11,12}$ to

Grant sponsor: National Science Council, Taiwan for JKH and CJL. *Correspondence to: Jenn-Kang Hwang, Department of Biological Science and Technology, National Chiao Tung University, Hsin Chu 300, Taiwan. E-mail: jkhwang@cc.nctu.edu.tw or Chih-Jen Lin, Department of Computer Science, National Taiwan University, Taipei, Taiwan. E-mail: lincj@NTU.edu.tw

Received 26 July 2002; Accepted 18 September 2002 
extract structural or physicochemical properties from the primary sequences, compressing 20 amino acids into three groups for the following attributes: the percentage composition of amino acids, predicted secondary structure, normalized van der Waals volumes, hydrophobicity, polarity, and polarizability. They then calculated three descriptors (i.e., "composition," "transition," and "distribution") for each attribute in these three groups of amino acids. Their approach yielded around 56.0\% prediction accuracy for an independent set. Despite seemingly lower prediction accuracy than before, the prediction was made in the context of 27 fine-grained SCOP folds, about one order higher than the number of protein classes used in their earlier work. They achieved this with a multiclass fold prediction system based on the jury votes from several parameter sets of structural or physicochemical properties of the sequences described by three groups of amino acids. In our work, using SVM coupled with more comprehensive protein descriptors based on $n$-peptide coding schemes and jury voting procedures, we can obtain a prediction accuracy significantly higher than that in the previously mentioned study.

\section{METHODS}

The SVM is a powerful classification method ${ }^{18}$ that has become popular in computational biology ${ }^{13,19-21}$ and other areas. The original idea of SVM is to use a linear hyperplane to separate training data in two classes: Given training vectors $x_{i}, i=1, \ldots, l$ and a vector $y$ defined as $y_{i}=$ 1 if $x_{i}$ is in one class, and $y_{i}=-1$ if $x_{i}$ is in the other class. The support vector technique tries to find the separating hyperplane $w^{T} x_{i}+b=0$, with the largest distance between two classes measured along a line perpendicular to this hyperplane. This requirement is equivalent to the minimization of $\frac{1}{2} w^{T} w$ with respect to $w$ and $b$ under the constraint that $y_{i}\left(w^{T} x_{i}+b\right) \geq 1$. However, in practice, these data to be classified may not be linearly separable. To overcome this difficulty, SVM nonlinearly transforms the original input space into a higher dimensional feature space by $\phi(x)=\left[\phi_{1}(x), \phi_{2}(x), \ldots\right]$ and tries to minimize

$$
\frac{1}{2} w^{T} w+C \sum_{i=1}^{l} \xi_{i}
$$

with respect to $w, b$, and $\xi$, under the constraint that $y_{i}\left(w^{T}\right.$ $\left.\phi\left(x_{i}\right)+b\right) \geq 1-\xi_{i}$, where $\xi_{i} \geq 0$. This procedure has the advantage of allowing training errors. It should be noted that only some of the $x_{i}$ 's are used to construct $w$ and $b$, and these data are called support vectors.

\section{Data Sets and Input Coding Schemes}

We used the same data set as that of Ding and Dubchak, ${ }^{13}$ which consists of 386 proteins of the most populated 27 SCOP folds in which the protein pairs have sequence identity below $35 \%$ for the aligned subsequences longer than 80 residues. These 27 proteins folds cover most major structural classes such as $\alpha, \beta, \alpha / \beta$, and $\alpha+\beta,{ }^{22}$ and have at least 7 or more proteins in their classes. To apply the machine learning techniques successfully to the bio- logic problems, one needs to extract relevant input vectors from the biologic data (i.e., the primary sequences). In this work, our global sequence-coding schemes cover the distribution of $n$-peptides for protein attributes. When $n$ is 1 , it encodes the composition of amino acids, which has been useful in discriminating the coarse-grained fold classes. ${ }^{14-16,23}$ When $n$ is 2 , the input vector encodes the dipeptide composition, which has been successfully applied to predict in vivo stability of proteins. ${ }^{24}$ We can extend $n$ to 3 or more, but it becomes impractical even in the case of $n=3$ (the size of the input vectors becomes $8000)$. This can be overcome if we reduce the size of the input vectors by regrouping the amino acids into a smaller number of classes according to their physicochemical properties. In this work, we denote the coding schemes by $X$ if all 20 amino acids are used, $X^{\prime}$ when the amino acids are classified as four groups-charged, polar, aromatic, and nonpolar-and $X^{\prime \prime}$, if predicted secondary structures are used. We assign the symbol $X$ the values of $\mathrm{D}, \mathrm{T}, \mathrm{Q}$, and $\mathrm{P}$, denoting the distributions of dipeptides, 3-peptides, and 4-peptides, respectively. Similar ideas that make use of $n$-gram models have been successfully applied to protein family identification. ${ }^{25}$ Because these parameters are built independently, one can apply machine learning techniques based on a single set of input vectors or a combination of several sets. All the SVM calculations are performed with LIBSVM, ${ }^{26}$ a general library for support vector classification and regression. We used PREDATOR ${ }^{27}$ to predict the secondary structure of the protein sequences.

\section{Training and Testing Procedures}

To have SVM classifiers perform a multiclass prediction, we followed two commonly used approaches. ${ }^{13}$ In the first, the "one-against-all" method, $F$ SVM classifiers are constructed and the $i$ th SVM is trained with proteins in the $i$ th fold as positive, and all other proteins as negative. Each protein in the test set is tested by all classifiers, and if positive, it will get a vote for the class. However, if it tests negative, this protein will not get any vote for the class. The "one-against-all" method gives rise to the possibility of giving some proteins too few or even no votes for any fold. However, we can complement this with the second method, "one-against-one," which is described as follows: Given $F$ classes of proteins, we can construct $F(F-1) / 2$ SVM classifiers and train with proteins from two different folds [in this work, we constructed for 27 folds a total of 27(27$1) / 2=351$ classifiers]. In this way, each protein in the test set will always get a vote for either one of the two folds, and the final assignment of folds to each protein in the test set is determined by the jury voting. Figure 1 shows the architecture of our SVM classifier. We use the standard $Q_{i}$ percentage accuracy ${ }^{13,28,29}$ for assessing the accuracy of protein fold identification $Q_{i}=c_{i} / n_{i} \times 100$, where $n_{i}$ is the number of test data in the $i$ th class and $c_{i}$ is the number correctly predicted. The overall $Q$ is given by

$$
Q=\sum_{i}^{F} w_{i} Q_{i},
$$




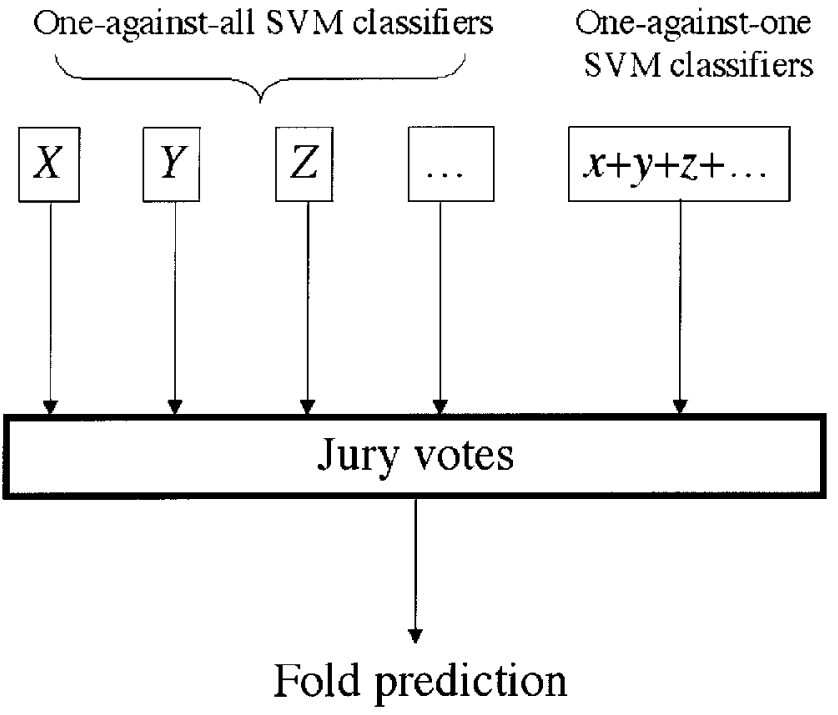

Fig. 1. The architecture of our SVM classifiers to predict the folds. The symbols $X, Y, Z, \ldots$ designate the parameter sets used in the "one-againstall" classifiers, and the symbols $x, y, z, \ldots$ the parameter sets used in the "one-against-one" classifiers. Each classifier casts one jury vote, and the fold that gets the most votes is the predicted fold for the query sequence.

where $w=n_{i} / N$, and $N$ is the total number of proteins.

We used two evaluation methods for the performance of the prediction system. First, we tested the system against the independent set, which comprised 385 proteins of 27 folds from the PDB-40D set ${ }^{30}$ that have sequence identity below $40 \%$ within the testing set, and below $35 \%$ compared with those of the training set. Second, we evaluated the classifiers by cross-validation, which measured their prediction accuracy systematically by first excluding a few proteins during the training process and then testing the classifiers against these excluded proteins. In the 10-fold cross-validation evaluation, each testing set comprised around $10 \%$ of the proteins. In addition to our parameter sets, we also used the following parameter sets of Dubchak et al. ${ }^{11,12}$ - the attributes of amino acids (C), predicted secondary structure $(\mathrm{S})$, and hydrophobicity $(\mathrm{H})$.

\section{RESULTS}

We compared the prediction accuracy of $n$-peptide coding schemes for the independent test set. Figure 2 gives the general trend of one-against-all prediction accuracies of isolated parameters sets: $X, X^{\prime}$, and $X^{\prime \prime}$. The parameter set $\mathrm{M}$, the composition of 20 amino acids $M$, gives the highest average prediction accuracy of 59\% for the 27 folds, as shown in Figure 2. The parameter D, the composition of dipeptides, gives much lower prediction accuracy. For the $X^{\prime}$ set, the composition of four classes of amino acids, the prediction accuracy displays the same monotonous decay when the length of the peptide fragments grows longer. It is interesting to note that $\mathrm{M}^{\prime}$ gives much lower prediction accuracy than $\mathrm{M}$, indicating that the composition of 20 amino acids contains more useful information in discriminating protein folds than the compressed classes of amino acids. For the $X^{\prime \prime}$ set, the composi- tion of predicted secondary structure, the prediction accuracy peaks at $\mathrm{D}^{\prime}$ and then slowly flattens out. To obtain the best overall prediction accuracy, we need a combination of parameters in both one-against-one and one-against-all classifiers. After some preliminary computations, we settled on the following parameter sets: M, D, $\mathrm{T}^{\prime}, \mathrm{Q}^{\prime}, \mathrm{P}^{\prime}$, and $\mathrm{T}^{\prime \prime}$ (using one-against-all classifiers), and $\mathrm{C}+\mathrm{S}+\mathrm{H}+\mathrm{D}$ (using one-against-one classifier), from which the highest combined votes will determine the predicted folds. Here C, S, and $\mathrm{H}$ are the percentage composition of amino acids, predicted secondary structure, and hydrophobicity, respectively. Table I lists our results for the independent set. In the one-against-one method, all the parameter sets $\left(\mathrm{M}, \mathrm{D}, \mathrm{T}^{\prime}, \mathrm{Q}^{\prime}, \mathrm{P}^{\prime}\right.$ and $\left.\mathrm{T}^{\prime \prime}\right)$ give average prediction accuracy greater than $40 \%$. In the one-against-one method, the parameter set $\mathrm{M}$, the composition of 20 amino acids, gives the best prediction accuracy of 59\% in the context of one parameter set (Fig. 2). Our results are consistent with previous findings ${ }^{14-16,23}$ that $\mathrm{M}$ is a very good discriminator in the classification of the coarse-grained folds. However, we also find that $M$, as an isolated parameter set, is also very helpful in identifying the 27 fine-grained classes of fold. The parameter sets $\mathrm{T}^{\prime}, \mathrm{Q}^{\prime}$, and $\mathrm{P}^{\prime}$ encode the distribution of tripeptide, 4-peptide, and 5-peptide sequences defined by amino acids that are classified into four groups. The parameter set $\mathrm{T}^{\prime}$ performs best, whereas $\mathrm{Q}^{\prime}$ and $\mathrm{P}^{\prime}$ give lower prediction accuracy. Among various combinations of parameter sets for the one-against-one method, we found that the $\mathrm{C}+\mathrm{S}+\mathrm{H}+\mathrm{D}$ set gave the best prediction accuracy at $63.1 \%$, which is higher than the one-against-all method using $\mathrm{M}$ set by around $4 \%$. The jury column in Table I gives the final prediction accuracy of $69.6 \%$ for each fold by the votes from the parameter sets, a $6.5 \%$ improvement on the one-against-one method, showing the effectiveness of the jury voting procedures..$^{13}$ In the breakdown analysis, our approach gives excellent prediction accuracy $(>80 \%)$ for the folds: $\alpha_{1}$ (globin-like $\alpha$-proteins), $\alpha_{2}$ (cytochrome $c$ folds), $\alpha_{5}$ (4-helical cytokines), $\beta_{1}$ (the immunoglobulin-like $\beta$-sandwich fold), $\beta_{7}$ (the trefoil fold), $(\alpha / \beta)_{1}$ (the triosephosphate isomerase (TIM)-barrel), and $(\alpha+\beta)_{3}$ (small proteins such as inhibitors, toxins, and lectins). On the other end of the prediction spectrum, our method gives poor results (accuracy $<50 \%$ ) for folds such as $\beta_{2}$ (cupredoxins), $\beta_{6}$ (oligonucleotide binding (OB)-fold), $(\alpha / \beta)_{3}$ (flavodoxin-like), $(\alpha / \beta)_{9}$ (periplasmic binding proteinlike) and $(\alpha+\beta)_{3}(\alpha+\beta)_{1}$ ( $\beta$-grasp or ubiquitin-like). These poor results reflect the consistent failure to recognize the correct folds by almost all the parameter sets. Figure 3 compares the prediction accuracy for each fold (in white) of our approach with that of Ding and Dubchak ${ }^{13}$ (in black). Our final prediction accuracy of $69.6 \%$ is a significant improvement on their result of $56.0 \%$ by $13.6 \%$. Our method gives better prediction for 24 folds, most noticeably $\alpha_{3}, \beta_{3}, \beta_{4}, \beta_{7}, \beta_{8}$, and $(\alpha+\beta)_{1}$, where improvements are more than 50\%. Both approaches give poor results for $\beta_{2}$ and $(\alpha / \beta)_{9}$. Figure 4 shows the 10 -fold cross-validation of the PDB-40D set, which was the result of randomly picking $10 \%$ of the protein as the test set during the training process and then testing the classifiers 


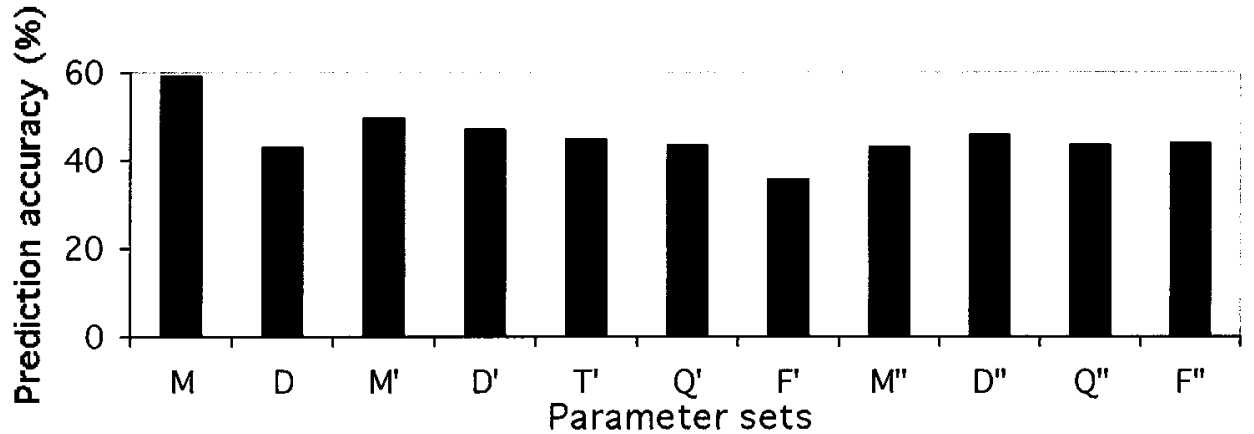

Fig. 2. Comparison of the "one-against-all" prediction accuracies of $X, X^{\prime}$, and $X^{\prime \prime}$ parameter sets. The symbols $\mathrm{M}, \mathrm{D}, \mathrm{T}, \mathrm{Q}$, and $\mathrm{F}$ represent $n$-peptide fragments with $n=1-5$, respectively.

TABLE I. Prediction Accuracy $Q_{i}(\%)$ for Protein Fold for the Independent Test Set

\begin{tabular}{|c|c|c|c|c|c|c|c|}
\hline \multirow[b]{2}{*}{ Folds $^{\mathrm{a}}$} & \multicolumn{5}{|c|}{ One-against-all } & \multicolumn{2}{|c|}{ One-against-one Jury } \\
\hline & $\mathrm{M}$ & $\mathrm{D}$ & $\mathrm{T}^{\prime}$ & $\mathrm{Q}^{\prime}$ & $\mathrm{T}^{\prime \prime}$ & $\mathrm{C}+\mathrm{S}+\mathrm{H}+\mathrm{D}$ & Final \\
\hline$\alpha_{1}$ & 83.3 & 83.3 & 66.7 & 100.0 & 66.7 & 83.3 & 83.3 \\
\hline$\alpha_{2}$ & 88.8 & 22.2 & 55.5 & 22.2 & 44.4 & 100.0 & 100.0 \\
\hline$\alpha_{3}$ & 55.0 & 30.0 & 55.0 & 40.0 & 40.0 & 40.0 & 70.0 \\
\hline$\alpha_{4}$ & 62.5 & 37.5 & 37.5 & 37.5 & 62.5 & 62.5 & 75.0 \\
\hline$\alpha_{5}^{4}$ & 100.0 & 66.7 & 55.5 & 44.4 & 66.7 & 100.0 & 100.0 \\
\hline$\alpha_{6}$ & 55.6 & 44.4 & 33.3 & 33.3 & 11.1 & 44.4 & 55.6 \\
\hline$\beta_{1}$ & 63.6 & 43.2 & 50.0 & 47.7 & 75.0 & 84.1 & 90.9 \\
\hline$\beta_{2}$ & 50.0 & 16.7 & 16.7 & 25.0 & 16.7 & 16.7 & 16.7 \\
\hline$\beta_{3}$ & 61.5 & 46.2 & 61.5 & 61.5 & 53.8 & 61.5 & 76.9 \\
\hline$\beta_{4}$ & 33.3 & 33.3 & 66.7 & 66.7 & 50.0 & 50.0 & 66.7 \\
\hline$\beta_{5}$ & 75.0 & 25.0 & 37.5 & 37.5 & 37.5 & 50.0 & 50.0 \\
\hline$\beta_{6}$ & 31.6 & 26.3 & 31.6 & 21.1 & 47.4 & 31.6 & 47.7 \\
\hline$\beta_{7}$ & 75.0 & 50.0 & 50.0 & 50.0 & 75.0 & 75.0 & 100.0 \\
\hline$\beta_{8}$ & 50.0 & 50.0 & 50.0 & 50.0 & 25.0 & 25.0 & 50.0 \\
\hline$\beta_{9}$ & 71.4 & 28.6 & 71.4 & 42.9 & 28.6 & 57.1 & 57.1 \\
\hline$(\alpha / \beta)_{1}$ & 83.3 & 66.7 & 60.4 & 62.5 & 45.8 & 87.5 & 93.8 \\
\hline$(\alpha / \beta)_{2}$ & 50.0 & 33.3 & 25.0 & 33.3 & 33.3 & 50.0 & 66.7 \\
\hline$(\alpha / \beta)_{3}$ & 30.8 & 7.7 & 15.4 & 30.8 & 15.4 & 53.8 & 38.5 \\
\hline$(\alpha / \beta)_{4}$ & 40.7 & 37.0 & 33.3 & 37.0 & 25.9 & 55.5 & 55.6 \\
\hline$(\alpha / \beta)_{5}$ & 50.0 & 33.3 & 41.7 & 33.3 & 33.3 & 50.0 & 50.0 \\
\hline$(\alpha / \beta)_{6}$ & 37.5 & 37.5 & 50.0 & 37.5 & 50.0 & 37.5 & 50.0 \\
\hline$(\alpha / \beta)_{7}$ & 42.9 & 42.9 & 42.9 & 42.9 & 42.9 & 57.1 & 57.1 \\
\hline$(\alpha / \beta)_{8}$ & 71.4 & 71.4 & 57.1 & 71.4 & 28.6 & 71.4 & 71.4 \\
\hline$(\alpha / \beta)_{9}$ & 25.0 & 25.0 & 50.0 & 50.0 & 25.0 & 25.0 & 25.0 \\
\hline$(\alpha+\beta)_{1}$ & 37.5 & 25.0 & 25.0 & 25.0 & 37.5 & 37.5 & 37.5 \\
\hline$(\alpha+\beta)_{2}$ & 22.2 & 22.2 & 25.9 & 18.5 & 25.9 & 48.1 & 51.9 \\
\hline$(\alpha+\beta)_{3}$ & 100.0 & 88.9 & 85.2 & 81.5 & 74.1 & 96.3 & 100.0 \\
\hline Avg & 59.0 & 43.1 & 47.0 & 44.9 & 44.9 & 63.1 & 69.6 \\
\hline
\end{tabular}

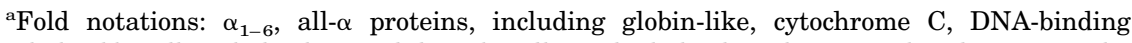
3-helical bundle, 4-helical up-and-down bundle, and 4-helical cytokines, EF-hand, respectively; $\beta_{1-9}$, all- $\beta$ proteins, including immunoglobulin-like $\beta$-sandwich, cupredoxins, viral coat and capsid proteins, ConA-like lectins/glucanases, SH3-like, barrel, OB-fold, $\beta$-trefoil, trypsin-like serine proteases, and lipocalins, respectively; $(\alpha / \beta)_{1-9}, \alpha / \beta$ proteins, including TIM-barrel, FAD/NAD-binding motif, flavodoxin-like NAD(P)-binding Rossmann-fold, P-loop-containing nucleotide, thioredoxin-like ribonuclease $\mathrm{H}$-like motif, hydrolases, and periplasmic binding protein-like, respectively; $(\alpha+\beta)_{1-3}, \alpha+\beta$ proteins, including $\beta$-Grasp, ferredoxin-like and small inhibitors, toxins, or lectins, respectively.

against the test sets. The results of cross-validation are consistent with those of the independent set. The final overall average prediction accuracy for the cross-validation is $65.3 \%$, which is also a significant improvement over the previous result of $45.4 \%$.

\section{DISCUSSION}

The previous work showed that in the coarse-grained fold assignment of major protein classes, such as all- $\alpha$, all- $\beta, \alpha+\beta, \alpha / \beta$ proteins, one could easily achieve high 


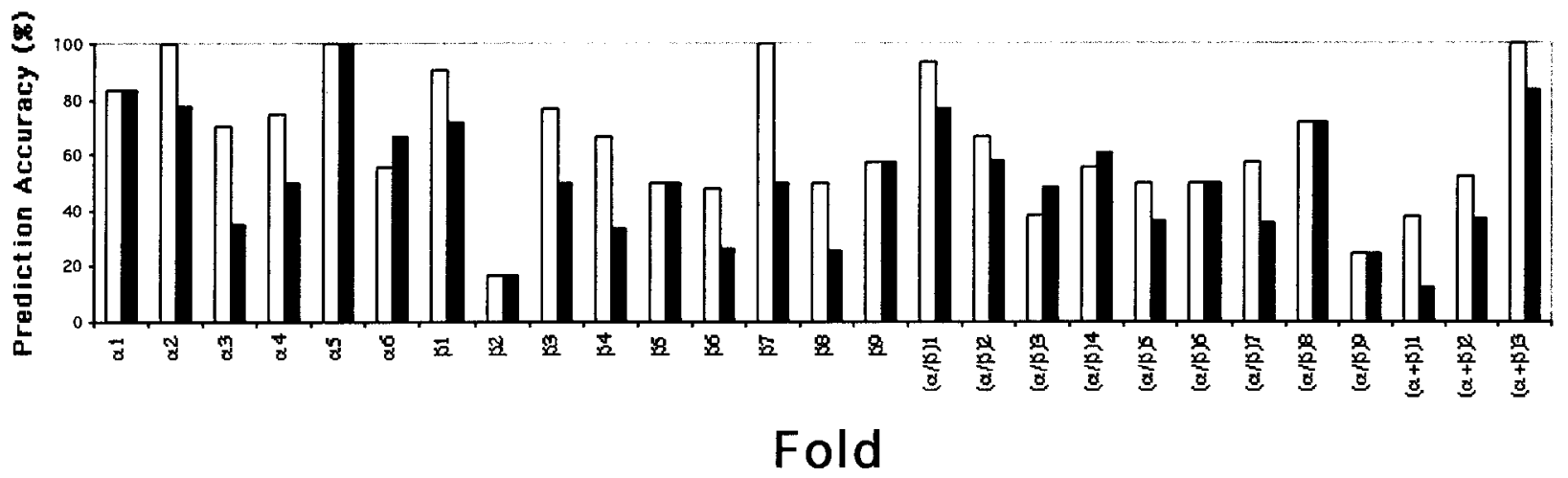

Fig. 3. Comparison of the prediction accuracy $Q_{i}(\%)$ of this work (in white) with that of Ding and Dubchak ${ }^{13}$ (in black) for the 27 folds in the independent test.

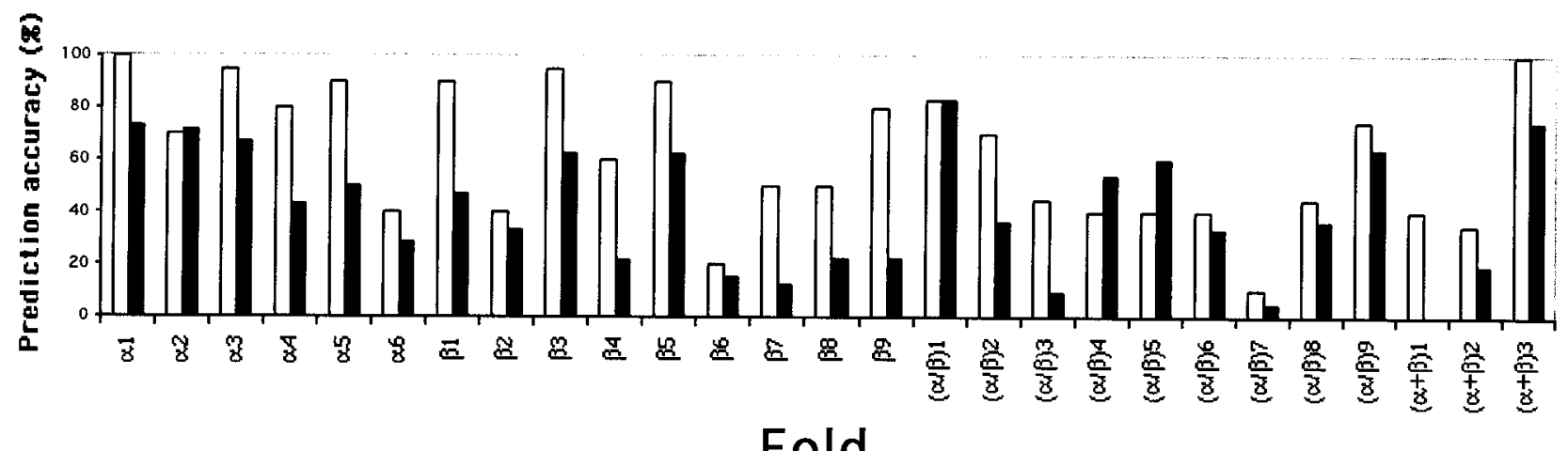

Fig. 4. Comparison of the prediction accuracy $Q_{i}(\%)$ of this work (in white) with that of Ding and Dubchak ${ }^{13}$ (in black) for the 27 folds in the 10 -fold cross-validation.

prediction accuracy (70-80\%) from amino acid composition. Ding and Dubchak ${ }^{13}$ showed that, in the fine-grained fold prediction, SVM combined with jury voting from multiple parameter sets yielded prediction accuracy significantly higher than that of any single parameter set: They obtained $56 \%$ prediction accuracy on an independent test set and $45.4 \%$ on cross- validation. We have demonstrated in this study that the amino acid composition $\mathrm{M}$ alone yield $59 \%$ prediction accuracy, which, though better than the current result, is still not yet practical in realistic applications. Using protein descriptors based on the properties derived from the composition of $n$-peptide and jury voting from a combination of parameter sets, we are able to achieve a $69.6 \%$ prediction accuracy on an independent set, an order of magnitude higher than the current results, and $65.3 \%$ on 10 -fold cross-validation. The prediction accuracy is approaching that for the coarse-grained fold classes. Our results show that SVM, novel, global sequencecoding schemes and proper combinations of input parameter sets should become an increasingly practical tool in structure modeling.

\section{REFERENCES}

1. Baker D, Sali A. Protein structure prediction and structural genomics. Science 2001;294:93-96.

2. Kihara D, Zhang Y, Lu H, Kolinski A, Skolnick J. Ab initio protein structure prediction on a genomic scale: Application to the Myco- plasma genitalium genome. Proc Natl Acad Sci U S A 2002;99: 5993-5998.

3. Xia Y, Levitt M, Huang ES, Samudrala R. Ab initio construction of protein tertiary structures using a hierarchical approach. J Mol Biol 2000;300:171-185.

4. Huang ES, Samudrala R, Ponder JW. Ab initio fold prediction of small helical proteins using distance geometry and knowledgebased scoring functions. J Mol Biol 1999;290:267-281.

5. Zhang CT, Hou J, Kim SH. Fold prediction of helical proteins using torsion angle dynamics and predicted restraints. Proc Natl Acad Sci U S A 2002;99:3581-3585.

6. Srinivasan R, Rose GD. Ab initio prediction of protein structure using LINUS. Proteins 2002;47:489-495.

7. Simons KT, Strauss C, Baker D. Prospects for ab initio protein structural genomics. J Mol Biol 2001;306:1191-1199.

8. Blundell TL, Sibanda BL, Sternberg MJ, Thornton JM. Knowledgebased prediction of protein structures and the design of novel molecules. Nature 1987;326:347-352.

9. Russell A, Torda AE. Protein sequence threading: Averaging over structures. Proteins 2002;47:496-505.

10. Kolinski A, Betancourt MR, Kihara D, Rotkiewicz P, Skolnick J. Generalized comparative modeling (GENECOMP): A combination of sequence comparison, threading, and lattice modeling for protein structure prediction and refinement. Proteins 2001;2001: 133-149.

11. Dubchak I, Muchnik I, Holbrook SR, Kim S-H. Prediction of protein folding class using global description of amino acid sequence. Proc Natl Acad Sci USA 1995;92:8700-8704.

12. Dubchak I, Muchnik I, Mayor C, Dralyuk I, Kim S-H. Recognition of a protein fold in the context of the structural classification of proteins (SCOP). Proteins 1999;35:401-407.

13. Ding CH, Dubchak I. Multi-class protein fold recognition using 
support vector machines and neural networks. Bioinformatics 2001;17:349-358.

14. Chou KC, Liu WM, Maggiora GM, Zhang CT. Prediction and classification of domain structural classes. Proteins 1998;31:97-103.

15. Chou KC, Zhang CT. Prediction of protein structural classes. Crit Rev Biochem Mol Biol 1995;30:275-349.

16. Dubchak I, Holbrook SR, Kim S-H. Prediction of protein folding class from amino acid composition. Proteins 1993;16:79-91.

17. Levitt M, Chothia C. Structural patterns in globular proteins. Nature 1976;261:552-558.

18. Vapnik V. The nature of statistical learning theory. New York: Springer; 1995.

19. Brown MP, Grundy WN, Lin D, Cristianini N, Sugnet CW, Furey TS, Ares M Jr, Hasussler D. Knowledge-based analysis of microarray gene expression data by using Support Vector Machine. Proc Natl Acad Sci U S A 2000;97:262-267.

20. Jaakkola T, Diekhans M, Haussler D. Using the Fisher kernel method to detect remote protein homologies. ISMB 1999;149-158.

21. Hua S, Sun Z. A novel method of protein secondary structure prediction with high segment overlap measure: Support vector machine approach. J Mol Biol 2001;308:397-407.

22. Murzin AG, Brenner SE, Hubbard T, Chothia C. SCOP: A structural classification of proteins database for the investigation of sequences and structures. J Mol Biol 1995;247:536-540.
23. Nakashima H, Nishikawa K, Ooi T. The folding type of a protein is relevant to the amino acid composition. J Biochem 1986;99:152162.

24. Guruprasad K, Reddy BVB, Pandit MW. Correlation between stability of a protein and its peptide composition: A novel approach for predicting in vivo stability of a protein from its primary sequence. Protein Eng 1990;4:155-161.

25. Wu CH, Zhao S, Chen HL, Lo CJ, McLarty J. Motif identification neural design for rapid and sensitive protein family search. Comput Appl Biosci 1996;12:109-118.

26. Chang CC, Lin CJ. LIBSVM: A library for support vector machines. 2001. Software available from http://www.csie.ntu.edu.tw/ $\sim$ cjlin/libsvm

27. Frishman D, Argos P. Knowledge-based secondary structure assignment. Proteins 1995;23:566-579.

28. Baldi P, Brunak S, Chauvin Y, Andersen C, Nielsen H. Assessing the accuracy of prediction algorithms for classification: An overview. Bioinformatics 2000;16:412-424.

29. Rost B, Sander C. Prediction of protein secondary structure at better than 70\% accuracy. J Mol Biol 1993;232:584-599.

30. Lo Conte L, Ailey B, Hubbard TJP, Brenner SE, Murzin AG, Chothia C. SCOP: A structural classification of protein database. Nucleic Acids Res 2000;28:257-259. 